\title{
Performance Analysis of Relative Positioning using GPS and BDS signals
}

\author{
Jae Hee $\mathrm{Noh}^{1}$, Sun Yong Lee ${ }^{2}$, Deok Won $\mathrm{Lim}^{3}$, Gwang Hee $\mathrm{Jo}^{1}$, Jin Hyuk Lee ${ }^{1}$ and Sang Jeong Lee ${ }^{1, *}$ \\ ${ }^{1}$ Department of Electronics Engineering, Chungnam National University, Daejeon 305-806, Republic of Korea \\ ${ }^{2}$ Navcours Co., Ltd., Daejeon 34014, Republic of Korea \\ ${ }^{3}$ Satellite Navigation Team, Korea Aerospace Research Institute, Daejeon 305-806, Republic of Korea
}

\begin{abstract}
In general, the satellite signal received by GNSS receivers has errors such as satellite clock error, orbit error, ionospheric delay and tropospheric delay. In environments where high positioning accuracy is required, these error factors can be eliminated by using relative positioning using code measurements with carrier phase measurements. If relative positioning is performed using carrier phase measurements, it is possible to have positioning accuracy of $\mathrm{cm}$ level. In this paper, we analyse the positioning accuracy of relative positioning using the L1 signal of GPS and BDS. For this study, we collect GPS and BDS signal using two low-cost receivers. We also designed a software-based platform to perform the relative positioning. Finally, we analyse relative positioning accuracy for GPS/BDS integrated system as well as relative positioning accuracy for GPS and BDS.
\end{abstract}

\section{Introduction}

There have been reported a lot of researches on precision positioning using GNSS in many fields requiring accurate positioning information. In particular, many studies are being conducted to provide accurate positioning to improve the accuracy and reliability of Intelligent Transportation Systems(ITS) and autonomous vehicle technology. When the GNSS signal is transmitted from the satellite to the receiver, the positioning error occurs due to various factors. Satellite clock errors, orbit errors, ionospheric delay, tropospheric delay and multipath are the factors that make it difficult for a GNSS receiver to estimate an exact position. In case of absolute positioning, correction methods are used to reduce these errors. However, the accuracy of positioning is lower than that relative positioning using double-difference observations. When code measurements and carrier phase measurements are double differenced, those errors are removed. So, the precise relative positioning using double-difference observations is widely used in the fields that require the high accuracy of positioning. In the field of GNSS, there are many positioning studies using the integration of GPS and GLONASS. Precise relative positioning using BDS has been actively studied since BDS provided open services. Currently, GNSS that can observe the most the number of visible satellite for 24 hours in Korea is BDS. So, it is necessary to study precise relative positioning using BDS. BDS satellites transmit ranging signals based on Cod e Division Multiple Access(CDMA) principle, like GPS. BDS provides the similar parameter as GPS in order to caculate stellite position [1-2].
In this paper, we anlayse the perfromance of precise relative positioing of GPS-only, BDS-only and GPS/BDS integrated systme using GPS L1 signal and BDS B1 signal. For this study, we collect GPS L1 signal and BDS B1 signal using two u-blox EVK-M8T receivers and design software based platform to perform precise relative posisitoing.

\section{Precise Relative Positioning}

In section 2.1, we described the characteristic of the signal of BDS according to the three-phase development plan. In section 2.2, we described the method of precise relative positioing using GPS and BDS.

\subsection{BDS Signal}

BDS consist of the three-phase development plan. The Beidou-1 system provided the regional active service in 2000 using two GEO(Geostationary Orbit) satellites. The Beidou-2 system provides open service for the AsisPacific region by the end of 2012. The Beidou-2 constellation consists of three types of satellites: GEO, IGSO(Inclined Geosynchronous Orbit) and MEO (Medium Earth Orbit). The accuracy of positioning of the Beidou-2 system is optimized to $6 \mathrm{~m}$. Table 1 summarizes Beidou-2 satellites in operation. By 2020, the Beidou-3 system will provide global service. When complete in 2020 , it will have a constellation of 35 satellites to provide global coverage [3-4].

\footnotetext{
${ }^{*}$ Corresponding author: jhnoh3555@cnu.ac.kr
} 
Table 1. Satus of Beiodu-2

\begin{tabular}{|c|c|c|}
\hline PRN & Type & Launched Time \\
\hline $\mathrm{C} 01$ & GEO & $2010 / 01 / 16$ \\
\hline $\mathrm{C} 02$ & GEO & $2012 / 10 / 25$ \\
\hline $\mathrm{C} 03$ & GEO & $2010 / 06 / 02$ \\
\hline $\mathrm{C} 04$ & GEO & 2010/10/31 \\
\hline $\mathrm{C} 05$ & GEO & $2012 / 02 / 24$ \\
\hline $\mathrm{C} 06$ & IGSO & $2010 / 07 / 31$ \\
\hline $\mathrm{C} 07$ & IGSO & $2010 / 12 / 17$ \\
\hline $\mathrm{C} 08$ & IGSO & 2011/04/09 \\
\hline C09 & IGSO & $2011 / 07 / 26$ \\
\hline $\mathrm{C} 10$ & IGSO & $2011 / 12 / 01$ \\
\hline $\mathrm{C} 11$ & MEO & $2012 / 04 / 29$ \\
\hline $\mathrm{C} 12$ & MEO & $2012 / 04 / 29$ \\
\hline $\mathrm{C} 13$ & IGSO & $2016 / 03 / 29$ \\
\hline C14 & MEO & $2012 / 09 / 18$ \\
\hline $\mathrm{C} 17$ & GEO & $2016 / 06 / 12$ \\
\hline
\end{tabular}

Currently, Beiodu-2 satellites transmit B1(1561.098 $\mathrm{MHz})$ signal, B2(1207.140 MHz) signal and B3(1269.520 $\mathrm{MHz}$ ) signal on channel I and channel Q. Channel I is used for civil services and channel Q is used for military purpose. When complete in 2020, Beidou-3 satellites transmit five signals. Beidou-3 satellites retain B1 and B3 signal. However, the B2 signal will have been replaced by three new signals, which are modernized signals in the $\mathrm{B} 1 \mathrm{C}, \mathrm{B} 2 \mathrm{a}$ and $\mathrm{B} 2 \mathrm{~b}$ bands [3].

\subsection{Precise Relative Positioning}

\subsubsection{GPS only \& BDS only}

The method of precise relative positioning uses the both double-difference code measurements and doubledifference carrier phase measurements. When estimating the user position, it is possible to estimate an accurate position of cm-level using both code measurement and carrier phase measurement. In the short-baseline, the equation of double-difference code measurements and double-difference carrier phase measurements of GPS and BDS is Eq. (1-4) [5]. The equation of double-difference code measurements of GPS is

$$
\lambda_{L 1} \Delta \nabla \Phi_{L 1, r u}^{i j}=\Delta \nabla \rho_{L 1, r u}^{i j}+\lambda_{L 1} \Delta \nabla N_{L 1, r u}^{i j}+\Delta \nabla \epsilon_{\Phi_{L 1}, r u}^{i j}
$$

The equation of double-difference carrier measurements of GPS is

$$
\lambda_{B 1} \Delta \nabla \Phi_{B 1, r u}^{i j}=\Delta \nabla \rho_{B 1, r u}^{i j}+\lambda_{B 1} \Delta \nabla N_{B 1, r u}^{i j}+\Delta \nabla \epsilon_{\Phi_{B 1}, r u}^{i j} .
$$

The equation of double-difference code measurements of BDS is

$$
\Delta \nabla P_{L 1, r u}^{i j}=\Delta \nabla \rho_{L 1, r u}^{i j}+\Delta \nabla \epsilon_{\Phi_{L 1}, r u}^{i j}
$$

The equation of double-difference carrier measurements of BDS is

$$
\Delta \nabla P_{B 1, r u}^{i j}=\Delta \nabla \rho_{B 1, r u}^{i j}+\Delta \nabla \epsilon_{\Phi_{B 1}, r u}^{i j}
$$

$\epsilon$ is residual errors, $\lambda_{L 1}$ is the length of the GPS L1 signal for one wavelength, $\lambda_{B 1}$ is the length of the BDS $\mathrm{B} 1$ signal for one wavelength. $r$ and $u$ denote the reference station and user, $i$ and $j$ is denote satellites. Eq. (5) is linear model using double-difference observations [5].

$$
y=\left[\begin{array}{c}
\rho_{P} \\
\rho_{\Phi}
\end{array}\right]=\left[\begin{array}{cc}
G_{u}(t) & 0 \\
G_{u}(t) & \lambda I_{n-1}
\end{array}\right]\left[\begin{array}{c}
\tilde{x}(t) \\
N_{u}
\end{array}\right]+\epsilon
$$

where $\tilde{x}(t)$ is unknown variables in linear model. The parameter of unknown variables includes the receiver position and the receiver clock error. Define $\tilde{x}(t)$ as

$$
\tilde{x}(t)=\left[\begin{array}{llll}
x & y & z & \delta t
\end{array}\right] .
$$

\subsubsection{GPS/BDS}

Eq. (5-6) are the linear model of precise relative positioning for a single GNSS. In the GPS/BDS integrated system, there are five unknown variables because the receiver clock error for two GNSS are considered [5]. The linear model and unknown variables for the GPS/BDS integrated system are defined as

$$
\begin{gathered}
y=\left[\begin{array}{l}
\rho_{P, L 1} \\
\rho_{\Phi, L 1} \\
\rho_{P, B 1} \\
\rho_{\Phi, B 1}
\end{array}\right]=\left[\begin{array}{ccc}
G_{u, L 1}(t) & 0 & 0 \\
G_{u, L 1}(t) & \lambda_{L 1} & 0 \\
G_{u, B 1}(t) & 0 & 0 \\
G_{u, B 1}(t) & 0 & \lambda_{B 1}
\end{array}\right]\left[\begin{array}{c}
\widetilde{x}(t) \\
N_{u, L 1} \\
N_{u, B 1}
\end{array}\right]+\epsilon \\
\tilde{x}(t)=\left[\begin{array}{lllll}
x & y & z & \delta t_{G P S} & \delta t_{B D S}
\end{array}\right] .
\end{gathered}
$$




\section{Field Experiments}

In this paper, we used the NovAtel GPS-703-GGG antenna and two u-blox EVK-M8T receivers to collect GPS L1 and BDS B1 signals in a zero-baseline environment. The elevation mask angle is $10^{\circ}$. Fig. 1 shows the configuration of the field experiment.

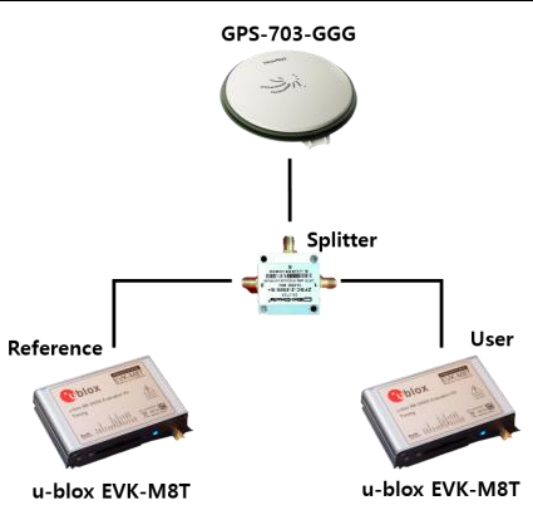

Fig. 1. Configuration of field experiment

We also designed software based platform to perform precise relative posisitoing of GPS-only, BDS-only and GPS/BDS integrated system. In section 3.1, we analyzed the visibility of GPS satellites and BDS satellites observed in the open sky for 24 hors. In section 3.2, based on results in section 3.1, we analyzed the accuracy of precise relative positiong when the number of visible satellite of GPS is the smallest. We also analyzed the accuracy of precise relative positiong when the number of visible satellite of BDS is the smallest.

\subsection{Satellite's visibility}

Fig. 2 shows the number of visible satellites of GPS and BDS observed for 24 hours in open sky. The elevation mask angle is $10^{\circ}$. For 24 hours, 6-12 GPS satellites can be observed and 11-14 BDS satellites can be observed. The result means that the number of visible satellites of BDS is more than that of visible satellite of GPS in Korea for 24 hours.

Fig. 3 shows the PDOP at 16:00 (UTC + 09) -17: 00 $(\mathrm{UTC}+09)$ when the number of visible satellite of GPS is the smallest. Fig. 4 shows the PDOP at the time of 20:30 $(\mathrm{UTC}+09)-21: 00(\mathrm{UTC}+09)$ when the number of visible satellite of BDS is the smallest. In Fig. (3-4), PDOP decreases when the integration of GPS and BDS. It can be seen that the geometrical arrangement of satellites is better for GPS/BDS integrated system than single

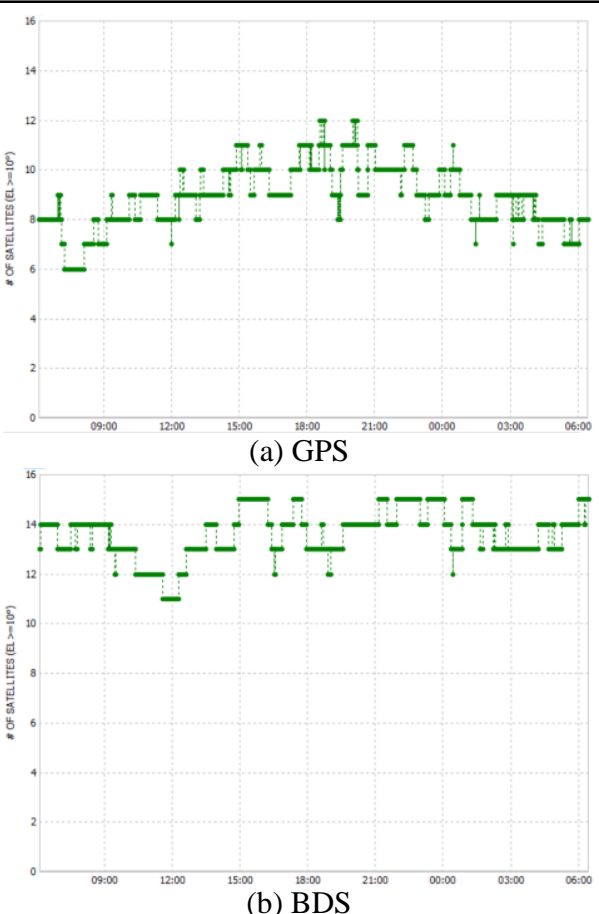

Fig. 2. The number of visible satellites of GPS and BDS for 24 hours

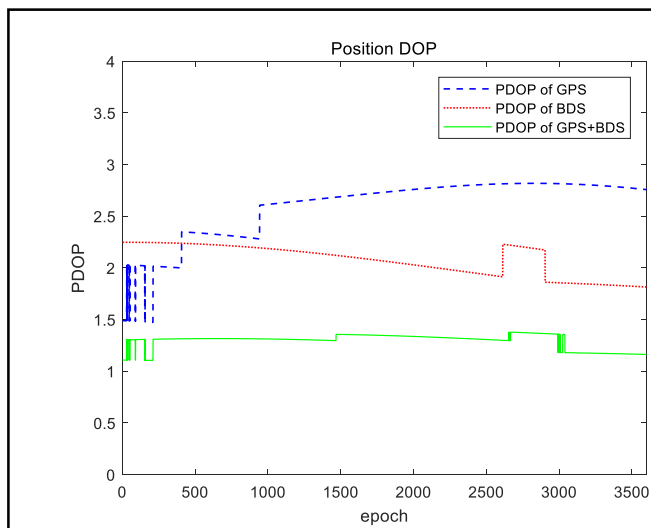

Fig. 3. PDOP at 16:00(UTC+09)-17:00(UTC+09)

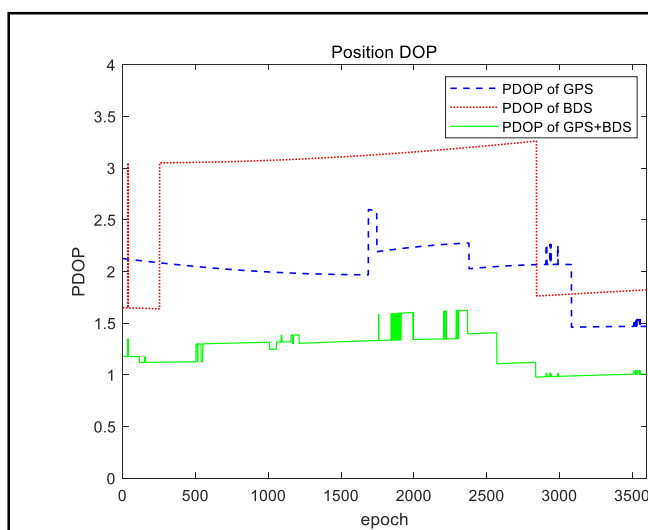

Fig. 4. $P D O P$ at 20:30(UTC +09$)-21: 30(U T C+09)$ 


\subsection{Results of experiments}

Fig. 5 shows the result of precise relative positioning of GPS-only, BDS-only and GPS / BDS integrated system at 16:00(UTC + 09)-17: 00(UTC + 09). At this time, the number of visible satellites of GPS is 6-7, and the number of visible satellites of BDS is 12-13.

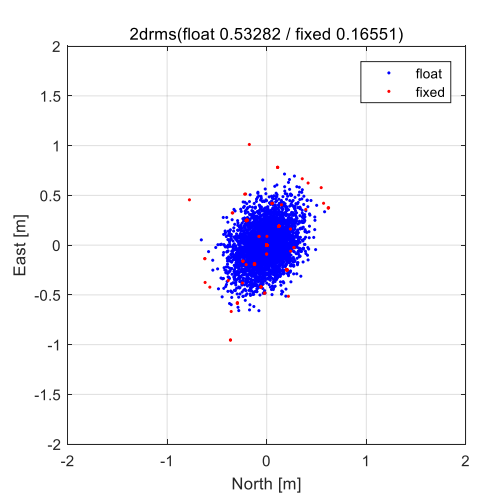

(a) GPS only

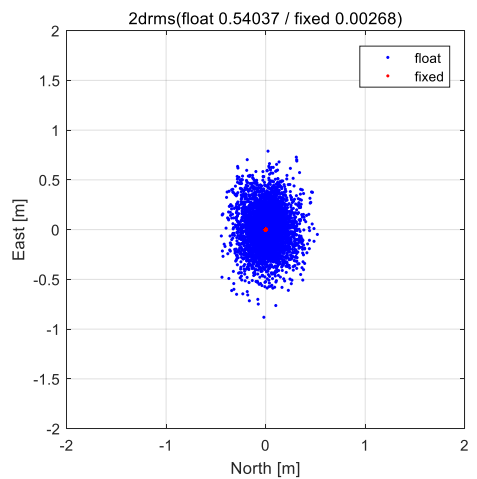

(b) BDS only

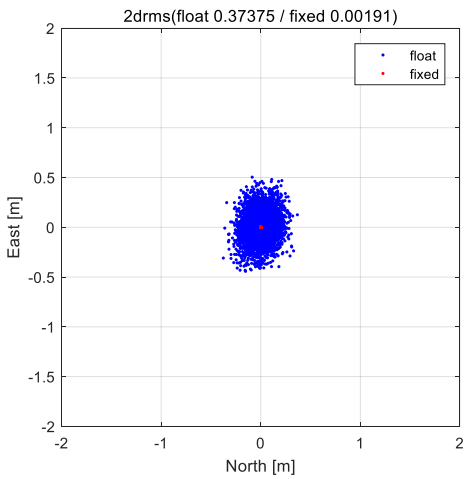

(c) GPS/BDS

Fig. 5. Results of precise relative positioing at $16: 00(\mathrm{UTC}+09)-17: 00(\mathrm{UTC}+09)$

The fixed solution of BDS-only is $2.7 \mathrm{~mm}$ and the fixed solution of GPS/BDS integrated system is $1.9 \mathrm{~mm}$. In the case of GPS-only, the fixed solution is $16.6 \mathrm{~cm}$. Because it couldn't observe enough visible satellites for precise relative positioning at 16:00 (UTC + 09) -17: 00 $(\mathrm{UTC}+09)$. The float solution of GPS-only and BDSonly is more than $45 \mathrm{~cm}$. However, the float solution of GPS/BDS integrated system is $37 \mathrm{~cm}$. This accuracy of positioning is below the maximum tolerance to distinguish the lane in the land traffic environment.
Fig. 6 shows the result of precise relative positioning of GPS-only, BDS-only and GPS / BDS integrated system at 20:30(UTC + 09)-21: 30(UTC + 09). At this time, the number of visible satellites of GPS is 810 , and the number of visible satellites of BDS is 11-12.

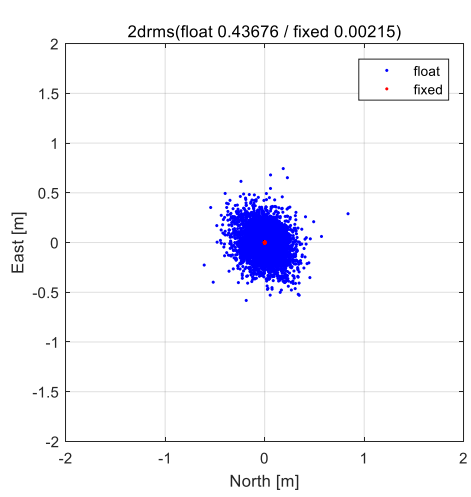

(a) GPS only

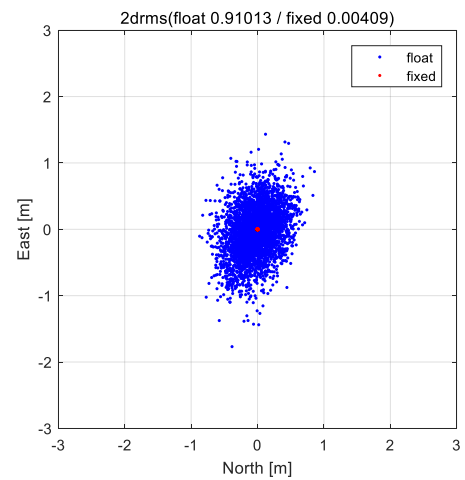

(b) BDS only

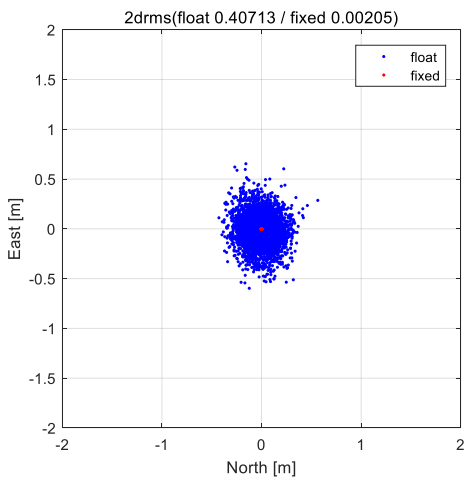

(c) GPS/BDS

Fig. 6. Results of precise relative positioing at $20: 30($ UTC+09)-21:30(UTC+09)

The fixed solution of GPS-only is $2.2 \mathrm{~mm}$. The fixed solution of BDS-only is $4.1 \mathrm{~mm}$. The fixed solution of GPS/BDS integrated system is $2.1 \mathrm{~mm}$. In all cases, the accuracy of positioning is mm-level. The float solution of BDS-only is more than $45 \mathrm{~cm}$. However, the float solution of GPS-only and GPS/BDS integrated system is below the maximum tolerance to distinguish the lane in the land traffic environment. 


\section{Conclusions}

In this paper, we analyzed the accuracy of precise relative positioning of GPS-only, BDS-only and GPS/BDS integrated system using GPS L1 signal and BDS B1 signal. Currently, the number of visible satellites of GPS is 6-12 for 24 hours in Korea. and the number of visible satellites of BDS is $11-15$ for 24 hours in Korea. It is possible to observe more BDS satellites than GPS satellites in Korea. When GPS and BDS are integrated, the number of satellites is increased, and the accuracy of positioing improves. As a result of precise relative positioing using GPS L1 signal and BDS B1 signal, the accuracy of positioing of GPS/BDS integrated system is more accurate than GPS-only and BDS-only. We analyzed the number of visible satellites of GPS and BDS in Korea for 24 hours and analyzed the performance of precise relative positioning. As a result, we can expect that BDS together with GPS is valuable for the research of precise positioining. However, it should be mentioned that the visibility of satellites can be not sufficient and evev worse multipath can occur in such an enviroment as urban canyon. So, it is necessary that analysis of the relative precision positioning using the GPS and BDS in the actual road environment.

\section{Acknowledgments}

This research was supported by a grant(18TLRPC113269-03) from Transportation \& Logistics Research Program funded by Ministry of Land, Infrastructure and Transport of Korean government.

\section{References}

1. Lee, J. M., Park, G. D., and Jeong, W. S., "Analysis of Positioning Accuracy for DGNSS-based Land Navigation Using GPS/BDS Navigation Satellite System," Transaction of KASE, 25, 4, 422-429 (2017)

2. Son, M. H., Son, E. S., Lee, E. S., Heo, M. B., and Nam, G. W., "Configuration of Network Based GNSS Correction System for Land Transportation Navigation," Journal of the Korean Society for Aviation and Aeronautics, 21, 4, 17-26 (2013)

3. Lu, X., "Construction and Development of Beidou Navigation Satellite System," in Committee on the Peaceful Uses of Outer Space, $61^{\text {st }}$ session, Austria, Vienna (2018)

4. International GNSS Service (Dec 8, 2018) available from : http://mgex.igs.org/IGS_MGEX_Status_BDS.php M.

5. Teunissen, P., Montenbruck, O., Springer Handbook of Global Navigation Satellite Systems, Switzerland : Spring Intenational Publishing (2017) 